\title{
Design equations for plates subjected to heat loads and lateral pressure
}

\author{
C. Guedes Soares*, J.M. Gordo, A.P. Teixeira \\ Unit of Marine Technology and Engineering, Instituto Superior Técnico, Universidade Técnica de Lisboa, \\ Av. Rovisco Pais, 1096 Lisboa, Portugal
}

Received 1 October 1999; received in revised form 26 November 1999; accepted 10 April 2000

\begin{abstract}
A brief description is presented of the strength behaviour of rectangular plates subjected to fire-induced intense heat loads of such a magnitude as to degrade the mechanical properties of the material. Design curves are proposed to predict the collapse of the plates under the biaxial state of stress induced by heat loads. Interaction curves are also derived for the case of plates that are initially subjected to lateral pressure and later to intense heat loads. (C) 2000 Elsevier Science Ltd. All rights reserved.
\end{abstract}

\section{Introduction}

Plate elements are one of the basic components of topsides of offshore platforms, while beam-columns are the components that make the framework supporting the platform topsides. Plate elements supported by the framework make the decks and ceilings. Plates are also used in the walls of the compartments, when they are aimed at providing blast resistance or only as a barrier for the thermal load induced by fires.

In both the horizontal and vertical components the plates can be stiffened. The understanding of the behaviour of the stiffened plates builds upon the knowledge of the behaviour of the unstiffened plate elements, which provide a significant strengthening effect to the associated stiffeners.

In a recent study [1], the authors have analysed the behaviour of steel plates subjected to thermal loads. The loading was a heat source that leads to a monotonically increasing temperature with uniform distribution in the plate. The

* Corresponding author. Tel.: 00351-1-841-7607; fax: 00351-1-847-4015.

E-mail address: guedess@alfa.ist.ult.pt (C. Guedes Soares). 
plate collapse was achieved by biaxial compression induced by the tendency of the plates to expand and the restrictions provided by the boundary conditions. It was concluded that the residual strength of the plate due to thermal loads is almost independent of the initial plate distortions and of the ambient temperature, i.e., the temperature of the surrounding structure.

Also, the normalised curves of average stress versus temperature agree particularly well in the post buckling region for the same plate geometry even for different types of steel. However, the curves are very different in the elastic region and the maximum carrying capacity is also different. It became apparent that the plate collapse load depends on the breadth-thickness ratio $(b / t)$. These observations suggest that design equations that predict the strength of plates under heat loads should include the dependence on this parameter.

This paper presents a brief description of the main characteristics of the collapse of plates under heat loads as a result of the biaxial state of stress that is developed. Design formulations that have been developed for plates under biaxial loading are reviewed and are used as a starting point to develop a design equation for plate collapse under heat loads.

The effect of heat load on plates subjected to lateral pressure are studied by means of a finite element code. Curves of stress versus temperature are produced for plates of different geometry under different levels of pressure.

Finally, interaction equations are derived for plates under lateral pressure and heat load.

\section{Plate collapse under heat loads}

For temperatures higher than $200^{\circ} \mathrm{C}$, the stress-strain characteristics of steels change by decreasing the yield stress and the modulus of elasticity. This effect combined with the increase of stresses associated with the temperature elevation leads to the collapse of plates. A review of the stress-strain properties of steels at elevated temperatures has been provided in [2] and the main recommendations were that, for current steels, the average curves specified in the Eurocodes [3] are the best option whenever there is no specific data for the steel under consideration.

Fig. 1 shows the dependence on temperature of the yield stress, the ultimate tensile stress and the Young's modulus (E), normalised by their values at ambient temperature. It is clear that while the yield stress and the Young's modulus start decreasing at temperatures of $100^{\circ} \mathrm{C}$, the ultimate tensile stress only starts to degrade at $400^{\circ} \mathrm{C}$.

At ambient temperature the collapse strength of plates is governed mainly by the plate slenderness, although the boundary conditions, the aspect ratio and the initial distortions are important parameters [4].

The load-shortening behaviour of plates with different aspect ratios, slenderness and initial distortions was determined by using a non-linear finite element code and keeping the boundary conditions of the plate restrained from linear displacements in its plane and calculating the corresponding edge reactions [1]. The loading was a heat source, leading to a monotonically increasing temperature with uniform distribution 


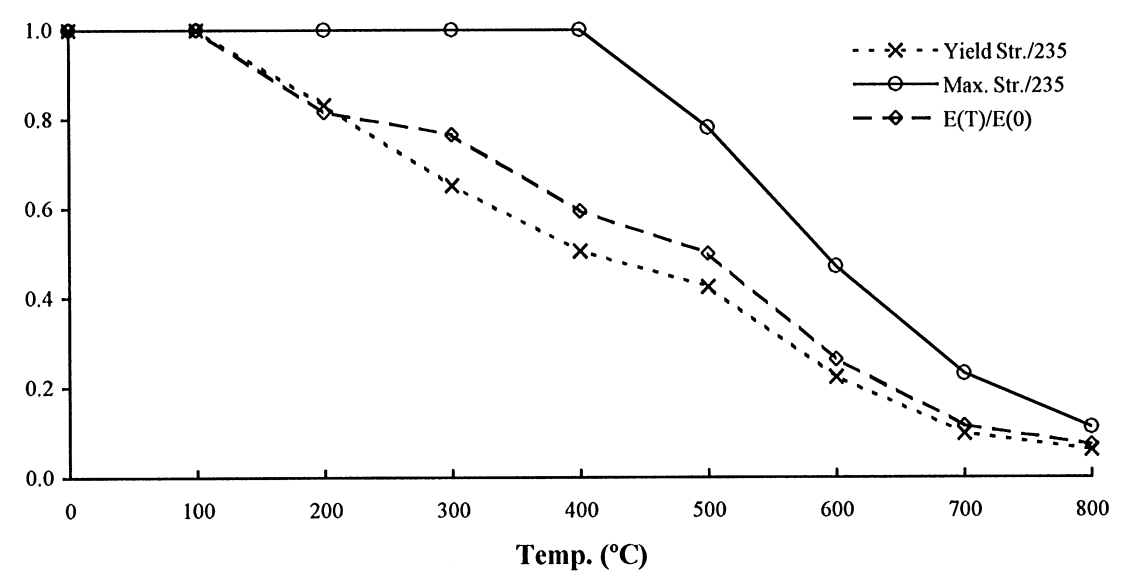

Fig. 1. Material properties of a mild steel [3].

in the plate, which varies from ambient temperature to values up to $800^{\circ} \mathrm{C}$. The assumption of uniform temperature in the plates results from the thermal conductivity of steel which leads to very quick heat conduction.

The numerical calculations were performed using the ASAS-NL code [5], which takes thermal loads into account. This is a general-purpose non-linear finite element code in which large displacement effects are handled using an updated Lagrangian formulation with inclusion of geometric stiffness terms for plate elements. The plasticity is modelled by the von Mises or Tresca yield criteria. The material behaviour can be non-linear and is defined by a piecewise linear, stress-strain curve for various temperatures. Properties at intermediate temperatures are obtained by linear interpolation.

To compute the element stiffness the material properties are updated according to the actual thermal load. The material stiffness can either be elastic or elasto-plastic depending whether plasticity has occurred at an integration point.

Having established that the thermal collapse load of plates is independent of their initial temperature, a series of calculations was conducted for several plates starting from an initial ambient temperature and subjected to a temperature increase up to collapse and continuing in the post-collapse range. The aspect ratios of the plates were 1 and 3 and the slenderness cover ranged from a $b / t$ of 20 to 100 .

The nominal plate slenderness is defined at ambient temperature as

$$
\beta=\frac{b}{t} \sqrt{\frac{\sigma_{o}}{E}},
$$

where $\sigma_{o}$ and $E$ are the yield stress and Young's modulus, respectively. However, when dealing with changing temperature, the definition of the plate slenderness presents a difficulty since the material properties of the plate are changing with 


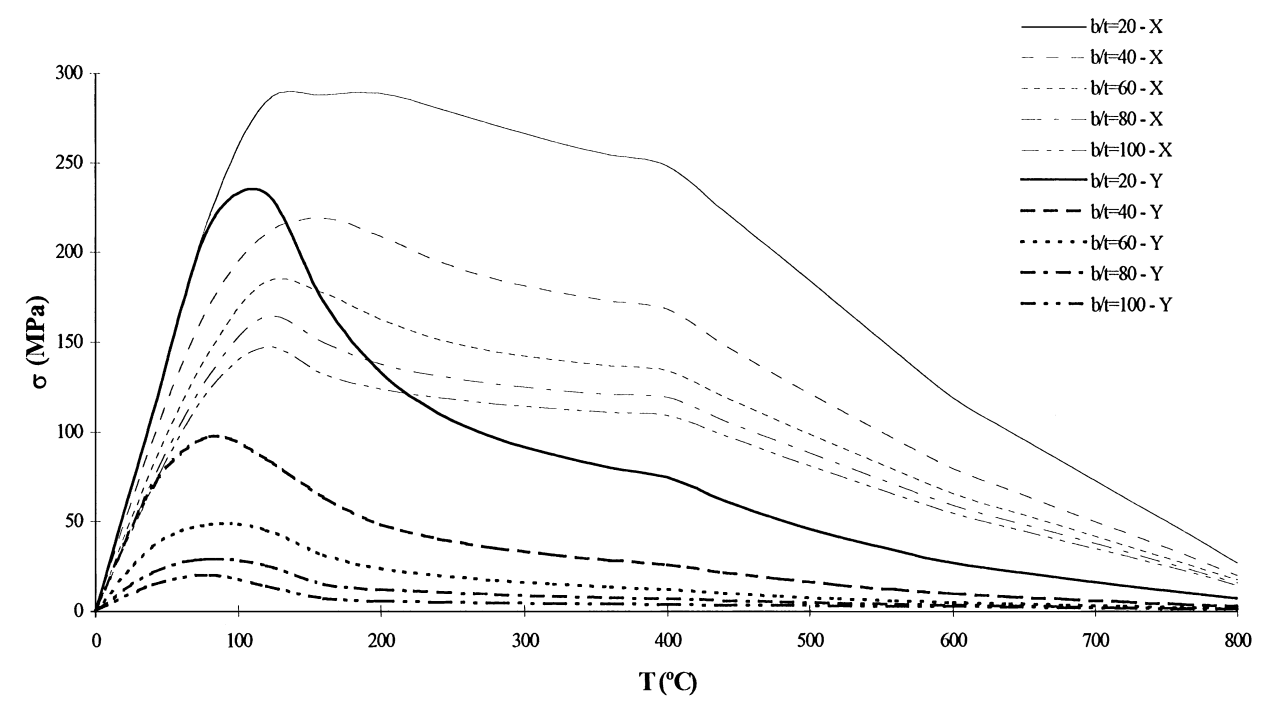

Fig. 2. Stress-temperature curves of rectangular plates $(a / b=3)$ of high strength steel with an average level of initial geometric imperfections $\delta / t=0.10 \beta^{2} ; X$ denotes the stresses in the longitudinal direction while $Y$ in the transverse one [1].

temperature. Thus, it is less meaningful to identify a plate with its slenderness at ambient temperature. In order to avoid this problem the plates are identified by their $b / t$ ratio.

The biaxial state of stresses is present in all plates especially in the elastic range $\left(T<100^{\circ} \mathrm{C}\right)$. Collapse in the transverse direction is achieved at a lower temperature and at lower stress levels, after which, the stresses in this direction fall quickly to very low levels while the longitudinal stresses keep increasing until the collapse is achieved. Fig. 2 presents a condensed view of the behaviour of rectangular plates under increasing temperature.

It was concluded [1] that for plates with aspect ratio different than one, with a predominant mode of imperfections equal to the length of the plate, the collapse in the transverse direction is achieved at lower temperatures $\left(T \approx 75^{\circ} \mathrm{C}\right)$ than the collapse in the longitudinal direction or the collapse of square plates $\left(T \approx 120^{\circ} \mathrm{C}\right)$ in which a failure occurs simultaneously in both directions.

\section{Design curves for plate collapse}

For design purposes including code specifications, several semi-empirical formulae have been proposed to predict the collapse strength of the plate elements subjected to predominantly compressive in-plane loads. Several of the expressions build upon the elastic buckling strength $\sigma_{c}$ of plate elements which is given in 
normalised form by:

$$
\phi=\frac{\sigma_{c}}{\sigma_{o}}=\frac{3.62}{\beta^{2}} .
$$

Expressions have been proposed with terms of this type but with other exponents and coefficients. Examples are the proposal of Faulkner [6]

$$
\phi_{F}=\frac{2}{\beta}-\frac{1}{\beta^{2}}
$$

or of Guedes Soares [4], who developed one which has the same starting point but deals explicitly with initial imperfections and residual stresses

$$
\begin{aligned}
\phi_{G S}= & \left\{1.08 \phi_{F}\right\}\left\{\left(1-\frac{\Delta \phi_{r}}{1.08 \phi_{F}}\right)(1-0.0078 \eta)\right\}\{(1-0.626-0.121 \beta) \delta\} \\
& \{0.665+0.006 \eta+0.36 \delta+0.14 \beta\},
\end{aligned}
$$

where $\Delta \phi_{r}$ is the strength reduction due to the existence of residual stresses $\sigma_{r}, \eta t$ is the width of the yield zone in tension on the edges of the plate, and $\delta$ is the magnitude of initial geometric distortions normalised by the plate thickness. The strength reduction is given by

$$
\Delta \phi_{r}=\frac{\sigma_{r}}{\sigma_{o}}\left(\frac{E_{t}}{E}\right)
$$

where

$$
\frac{\sigma_{r}}{\sigma_{o}}=\frac{2 \eta}{(b / t)-2 \eta}
$$

and $E_{t}$ is the tangent modulus given by [7]

$$
\begin{aligned}
& \frac{E_{t}}{E}=\frac{\beta-1}{1.5} \text { for } 1 \leqslant \beta \leqslant 2.5, \\
& \frac{E_{t}}{E}=1 \quad \text { for } \beta \geqslant 2.5 .
\end{aligned}
$$

The influence of biaxial stresses is dealt with by interaction curves which relate the longitudinal and transverse stresses. Different proposals exist in the literature but possibly the most commonly used is the circular interaction

$$
R_{x}^{2}+R_{y}^{2}=R^{2},
$$

where collapse is reached for $R=1$ and $R_{x}$ and $R_{y}$ are the ratios of the applied stress to the ultimate stress in each direction:

$$
\begin{aligned}
& R_{x}=\frac{\sigma}{\sigma_{u x}}, \\
& R_{y}=\frac{\sigma}{\sigma_{u y}} .
\end{aligned}
$$


The ultimate longitudinal stress can be defined by Eq. (3), $\sigma_{u x}=\phi_{u x} \sigma_{o}=\phi_{F} \sigma_{o}$, and the ultimate transverse stress by the equation due to Valsgard [8]

$$
\sigma_{u y} \equiv \phi_{u y} \sigma_{o}=\left[\frac{\phi_{u x}}{a / b}+0.08\left(1+\frac{1}{\beta^{2}}\right)^{2}\left(1-\frac{b}{a}\right)\right] \sigma_{o} .
$$

The von Mises curve is sometimes used for very stocky plates because the collapse of these plates is due to plasticity instead of buckling. In order to include buckling a generalised von Mises interaction may be introduced by replacing the yield stress by the ultimate stress in each direction. The von Mises curve is given by

$$
R_{x}^{2}+R_{y}^{2}-R_{x} R_{y}=R^{2} .
$$

More conservative is the parabolic interaction formula proposed by Faulkner et al. [9] which is given by

$$
R_{x}+R_{y}^{2}=R^{2}
$$

in which the transverse ultimate stress to be used to normalise the second term of the first member is the one proposed by Faulkner to predict the ultimate strength of plates under transverse loads [9].

These formulations have been reviewed by Guedes Soares and Gordo [10], who compared them with experimental evidence and numerical results, proposing a formulation to be adopted for prediction of the biaxial strength.

To check the adequacy of these formulations for the case of plate collapse under heat loads, calculations have been made applying the results of the computer runs [1] to these formulae. The calculated interaction ratio for square plates are plotted in Figs. 3 and 4 assuming the circular (Eq. (8)) and the von Mises interaction (Eq. (12)), both based on the effective width given by (Eq. (3)). The stress ratios are calculated considering the yield stress and the Young's modulus of the material for each temperature. For high temperatures, the yield stress may be half of the ultimate tensile stress and, consequently, the prediction will be on the conservative side since the material will be able to sustain the ultimate stress.

One may note that the first interaction is conservative by more than $20 \%$ for all $b / t$ ratios $(R \approx 1.2)$, while the second one is optimistic for $b / t>40(R<1.0)$. The plate of $b / t=20$ shows a very high strength when compared with the other curves which have a small spread. This should be due to the different mode of collapse which, in this case, is due to plasticity of the material while, in the other, buckling is the most important one. Because of this, the von Mises formula approaches one at this slenderness $(b / t=20)$.

All curves grow until a peak is reached at $600^{\circ} \mathrm{C}$ and after that point they decrease. This is due to the fact that the interaction and the strength prediction formulae are not being normalised by the value of the ultimate tensile stress of the material at each temperature but by the respective yield stress. When these curves are compared with the ratio between these two stresses (Fig. 1) the reason for this behaviour becomes evident. 


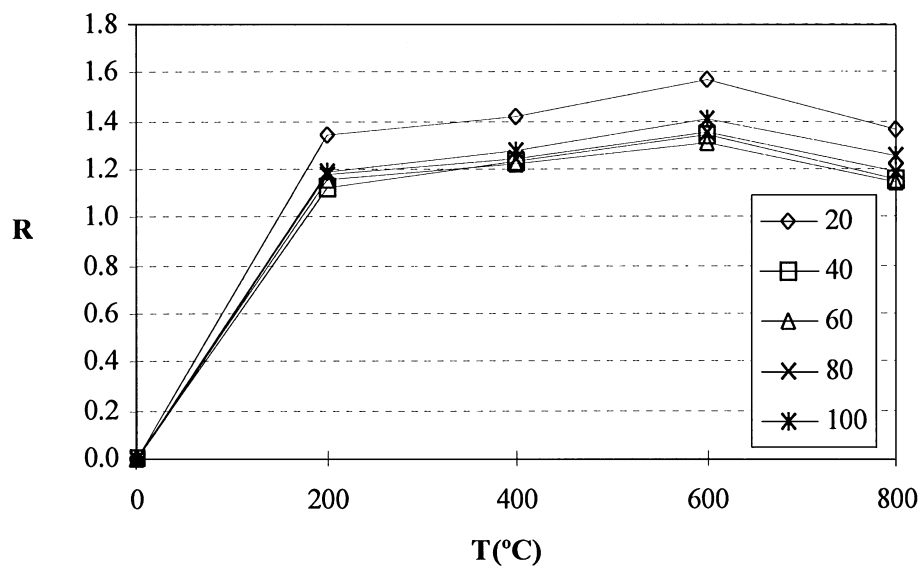

Fig. 3. Interaction ratio $(R)$ for the circular interaction formula applied to square plates of several $b / t$ ratios.

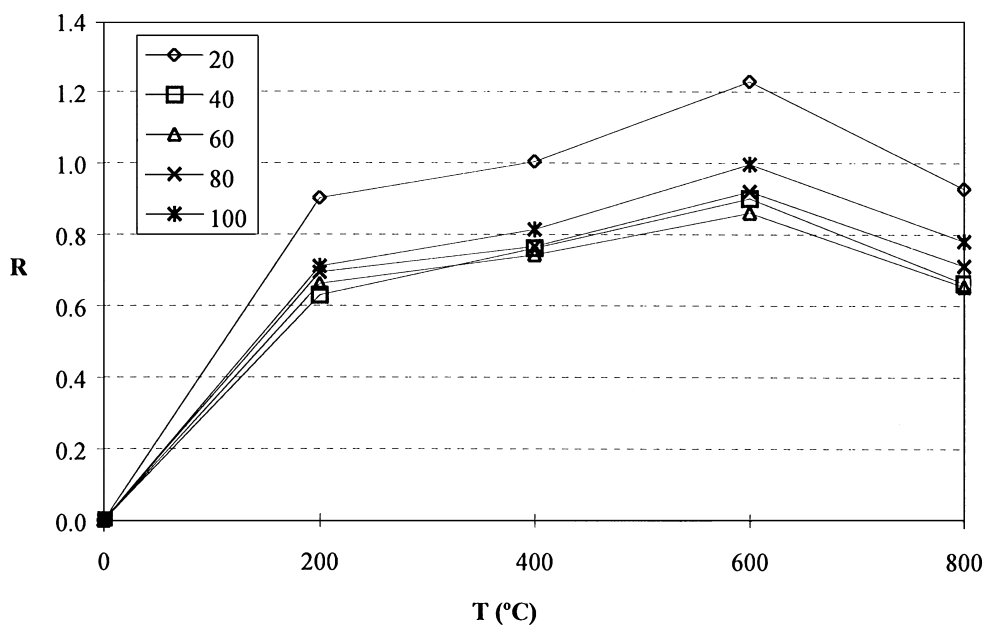

Fig. 4. Interaction ratio $(R)$ for the von Mises interaction formula applied to square plates of several $b / t$ ratios.

Figs. 5 and 6 show the interaction of plates with an aspect ratio of 3 with average initial imperfections in the first mode. The curves are similar to those of square plates but, now, the two interaction formulae give approximately the same results because the transverse stresses are very low due to the collapse of the plate in the first mode. Thus, the load supported by the plate is almost entirely longitudinal and for both interaction equations $R \approx R_{\mathrm{x}}$.

Fig. 7 shows the variation of the ratio between the transverse and the longitudinal stresses with temperature. These two stresses are strongly related and one can 


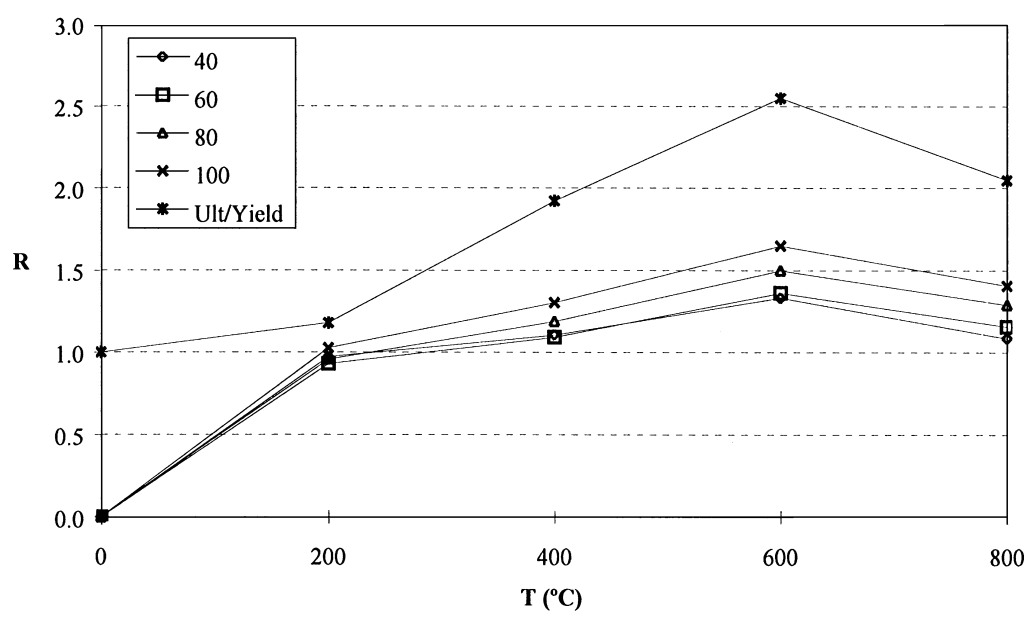

Fig. 5. Circular interaction formula applied to plates of several $b / t$ ratios and aspect ratio of 3 . The ratio between ultimate tensile stress and yield stress of the material at each temperature is also shown.

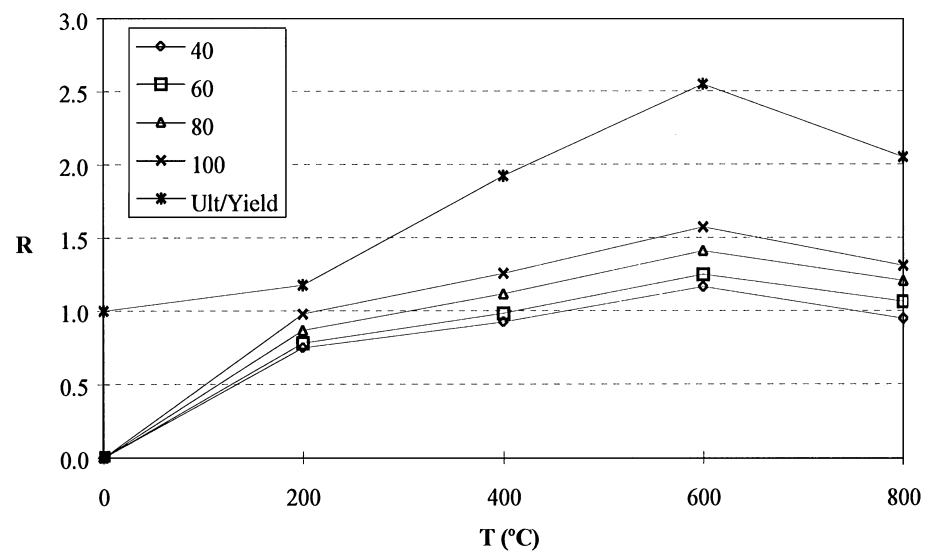

Fig. 6. von Mises interaction formula applied to plates of several $b / t$ ratios and aspect ratio of 3 . The ratio between ultimate tensile stress and yield stress of the material at each temperature is also shown.

conclude that the transverse stress may be estimated as a fraction of the longitudinal stress. The ratio varies between 15 and $5 \%$ in the range of interest, experiencing the highest values at low slenderness and decreasing with increasing slenderness. It is interesting to note the constancy of the ratio after the collapse of the plate $\left(T>180^{\circ} \mathrm{C}\right)$ in spite of the sharp decrease in strength with increasing temperature.

As the strength of the plates demonstrates a strong dependency upon the ultimate tensile stress of the material at each temperature, it is appropriate to include this 


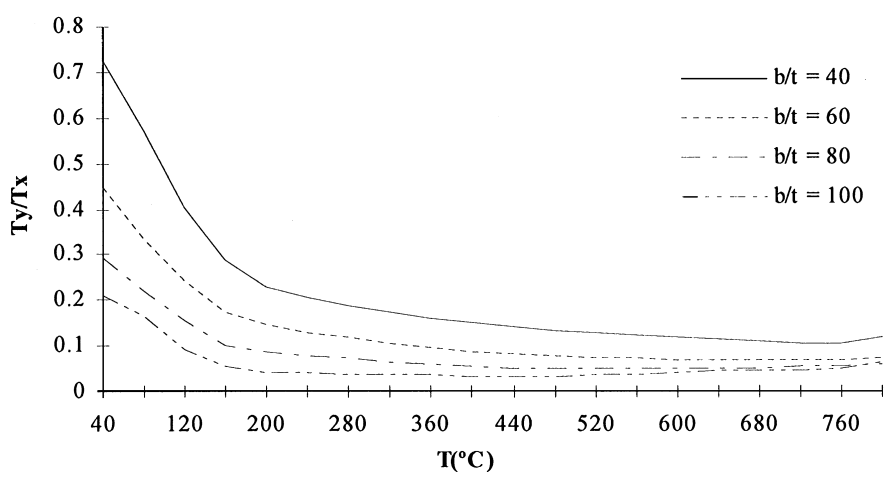

Fig. 7. Variation of the ratio between transverse and longitudinal average stresses normalised by the yield stress as function of temperature for plates with $a / b=3$.

parameter in the formulation for the prediction of the strength. To do this one should note that an average edge strain $\varepsilon_{a}$ might be associated with the actual temperature:

$$
\varepsilon_{a} \equiv \varepsilon(T)=\alpha_{t T} .
$$

If this temperature is sufficiently high then the corresponding strain is higher than the yield strain and the highest stress in the plate is higher than the yield stress. The highest stress may be read directly from the curves of material behaviour or estimated by a simple interpolation between the ultimate tensile stress $\left(\varepsilon_{m}, \sigma_{m}\right)$ and the yield stress $\left(\varepsilon_{o}, \sigma_{o}\right)$.

$$
\sigma_{a}=\sigma_{o}+\frac{\sigma_{m}-\sigma_{o}}{\varepsilon_{m}-\varepsilon_{o}}\left(\varepsilon_{a}-\varepsilon_{o}\right)
$$

This second process calculates a stress lower than the one that is read directly from the plots but the approximation is good enough. The design formulae can now be checked in the same way, as described before, but using the actual stress, $\sigma_{a}$, instead of the yield stress. Figs. 8 and 9 plot the interaction ratios for the plates using the circular interaction Eq. (8) for aspect ratios of 1 and 3, respectively. The results can be seen to have improved significantly compared with those that were not normalised by the highest stress present in the plate $\left(\sigma_{a}\right)$. The skew with temperature has been almost eliminated, especially in the range below $600^{\circ} \mathrm{C}$. The mean value and the coefficient of variation $(\mathrm{COV})$ of the data is reduced to acceptable values: 0.91 and 1.01 for the mean value and 12.2 and $11.8 \%$ for the COV, respectively, for aspect ratios of 1 and 3 .

Similar calculations were also performed using the von Mises interaction. The results obtained are illustrated in Figs. 10 and 11 showing that this design equation is much more conservative especially for square plates. The mean value of the data is now 0.65 and 0.92 , respectively, for aspect ratios of 1 and 3 . 


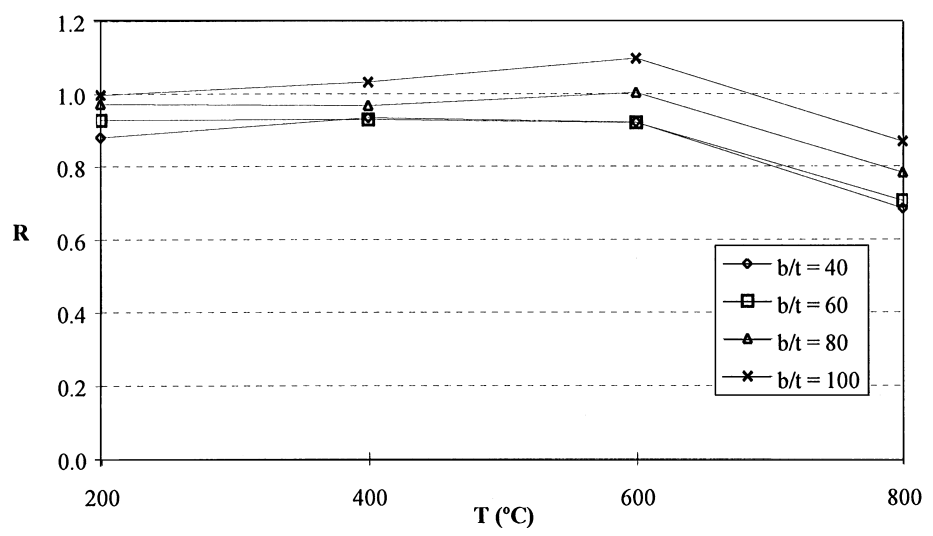

Fig. 8. Circular interaction formula applied to square plates of several $b / t$ ratios. The stress ratios are estimated using the actual material properties.

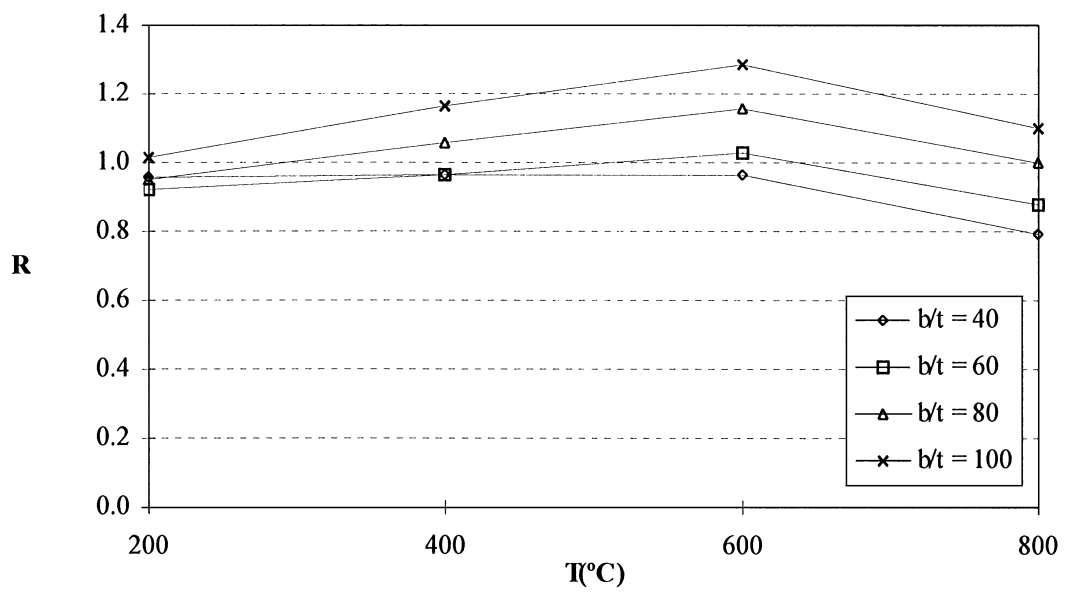

Fig. 9. Circular interaction formula applied to plates of several $b / t$ ratios and aspect ratio of 3 . The stress ratios are estimated using the actual material properties.

Figs. 8 and 9 allows one to conclude that the adoption of the circular interaction with the stresses normalised by the ultimate stress of the material at the corresponding temperature constitutes a good design equation, as $R$ is very close to unity along the range of temperatures.

\section{Influence of lateral pressure on plate behaviour}

The effect of lateral pressure was investigated for square plates and rectangular plates of aspect ratio 3. The applied lateral pressure was uniformly distributed on 


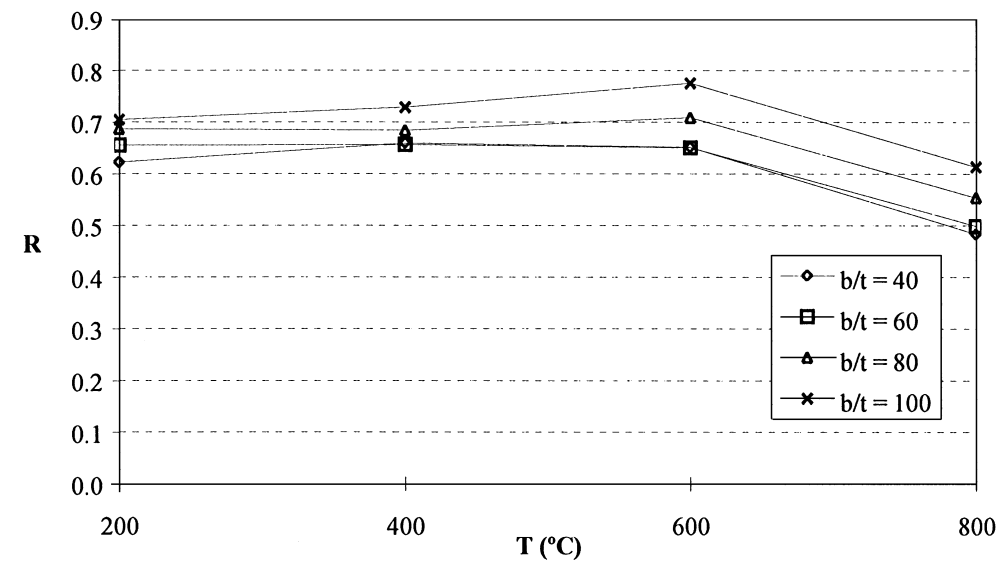

Fig. 10. von Mises interaction formula applied to square plates of several $b / t$ ratios. The stress ratios are estimated using the actual material properties.

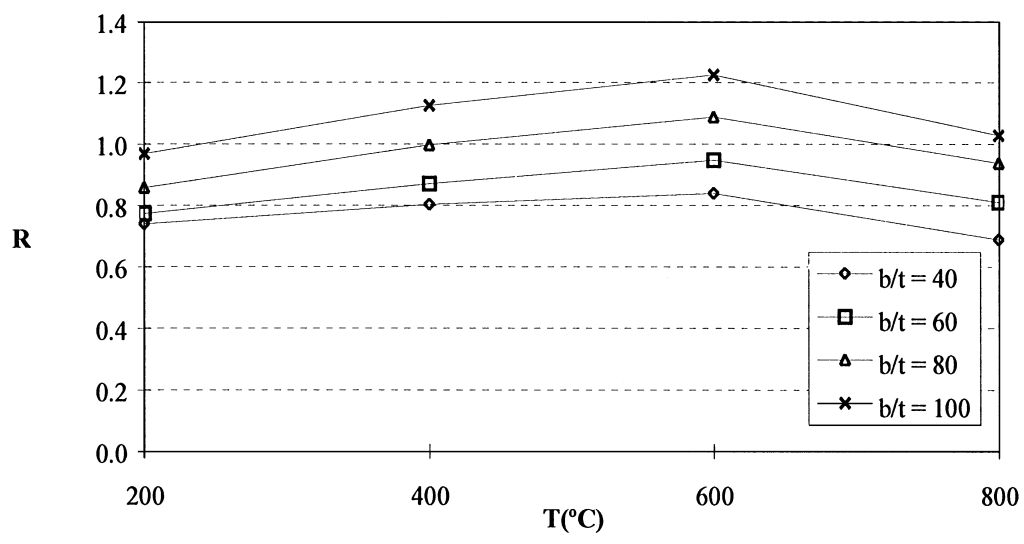

Fig. 11. von Mises interaction formula applied to plates of several $b / t$ ratios and aspect ratio of 3 . The stress ratios are estimated using the actual material properties.

simply supported plates with restrained edges to linear displacements in its plane. Five levels of plate slenderness were considered with corresponding width-thickness ratio of $20,40,60,80$ and 100 .

An average level of initial geometric imperfections $\delta_{a}$, was considered for both square and rectangular plates

$$
\delta_{a} / t=0.10 \beta^{2},
$$

where $\beta$ is the plate slenderness at ambient temperature. 
The shape of the initial geometric imperfections is represented by:

$$
w=\sum_{m} \sum_{n} \delta_{m n} \sin \frac{m \pi x}{a} \sin \frac{n \pi y}{b}
$$

where $a$ and $b$ are the plate dimensions and $\delta_{m n}$ is the amplitude of the components. In each calculation the initial distortion of the plate was represented by a shape with only one component of this series $(m=1 ; n=1)$.

The procedure used to account for lateral pressure consists of initially applying the pressure and then subsequently increasing the temperature. This loading sequence aims at modelling a situation of a structure, such as a deck, supporting some lateral load which is then subjected to a fire load that induces a temperature increase.

The calculations were performed using the ASAS_NL finite-element program described briefly in Section 2.

\subsection{Behaviour of square plates}

Figs. 12 and 13 show the plate behaviour for a lateral pressure of 0.1 and $0.5 \mathrm{MPa}$, respectively. It can be observed that initially the plates are in tension due to the effect of the lateral pressure but the increasing temperature and the corresponding expansion restrained by the fixed edges, tend to create a compression stress state in the plates.

However, very slender plates $(b / t=100)$ loaded with a high pressure do not experience compression stresses because the thermal expansion is compensated by the large out-of-plane deformations due to the pressure loading which decrease the

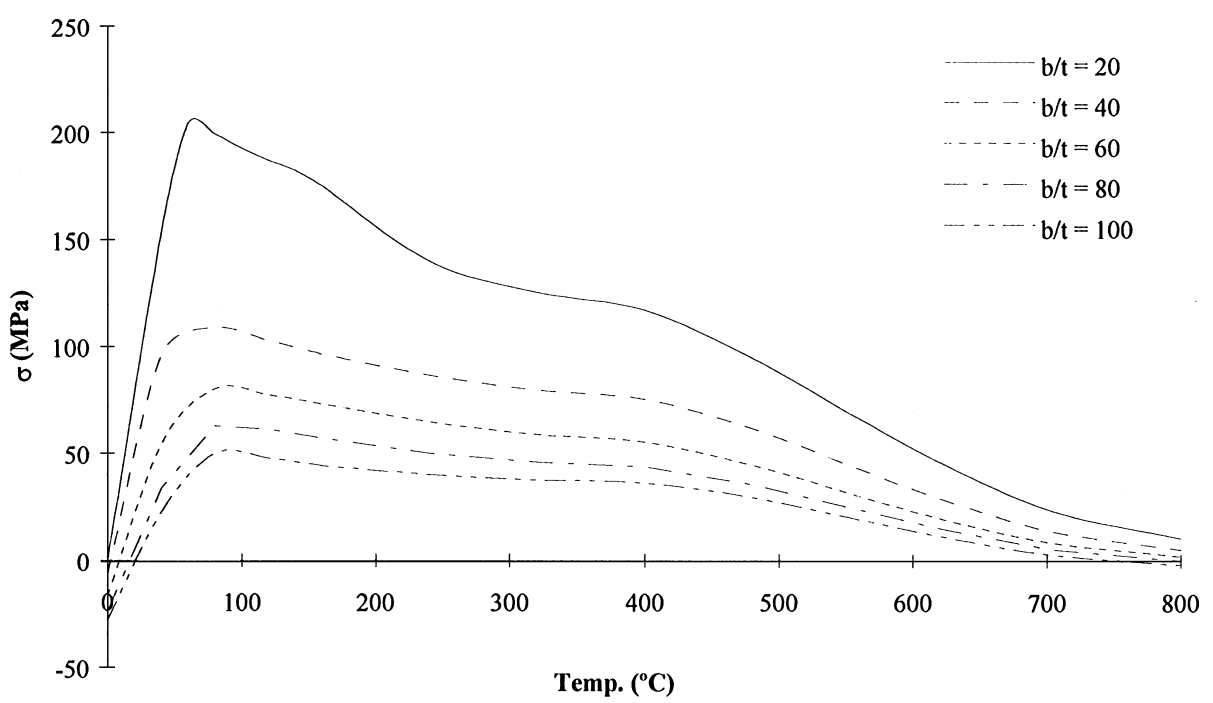

Fig. 12. Behaviour of square plates under temperature with applied lateral pressure of $0.1 \mathrm{MPa}$. 


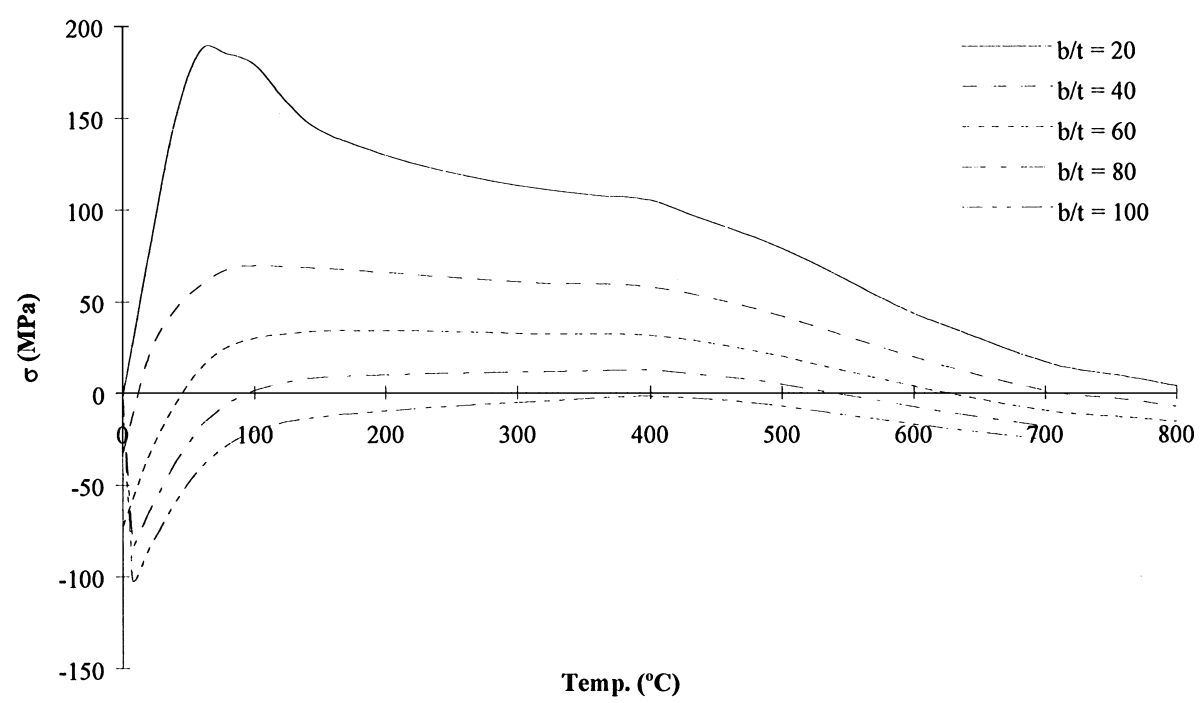

Fig. 13. Behaviour of square plates under temperature with applied lateral pressure of $0.5 \mathrm{MPa}$.

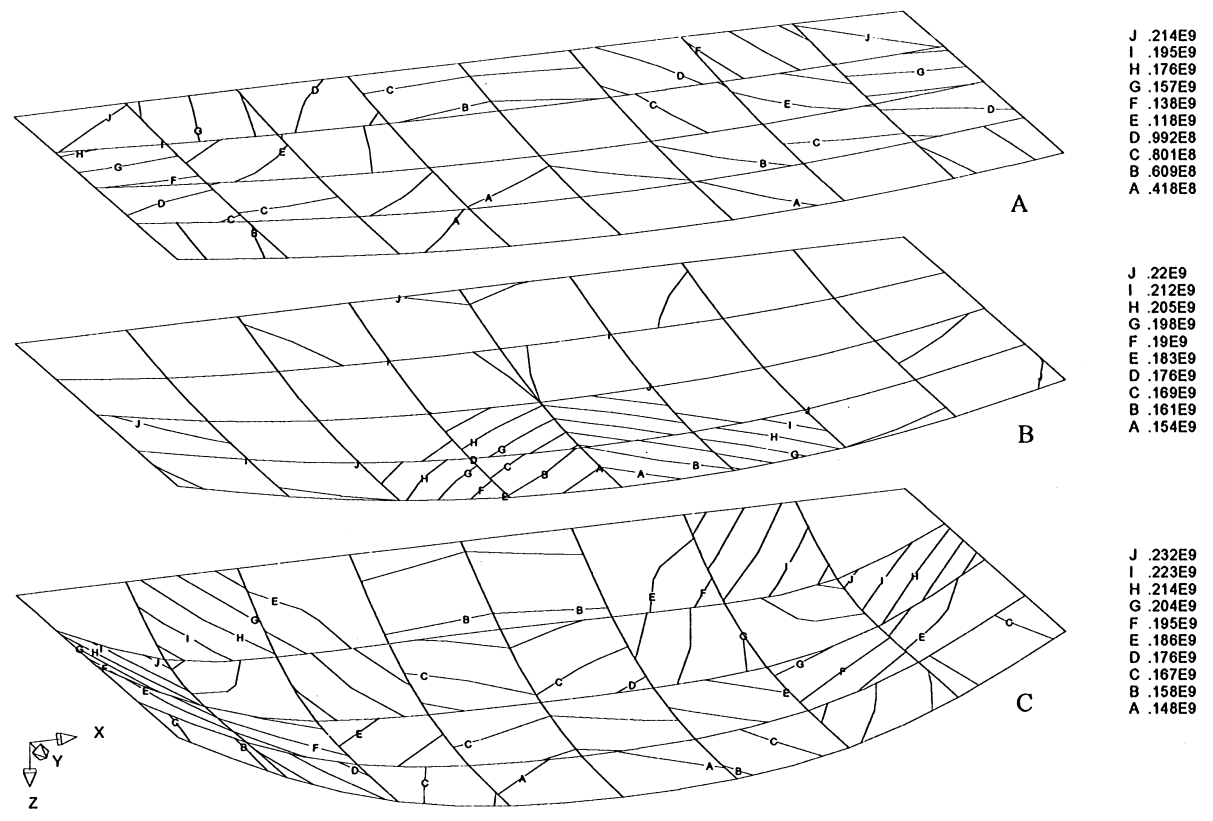

Fig. 14. Combined stress distribution for three different temperature levels of square plate $(b / t=60)$ with applied lateral pressure of $0.1 \mathrm{MPa}$ (A) $0^{\circ} \mathrm{C}$, (B) $100^{\circ} \mathrm{C}$, (C) $400^{\circ} \mathrm{C}$. 
average compressive stress. In spite of these low levels of stresses, square plates of high slenderness show very large deformations when the temperature is associated with lateral pressure.

Fig. 14 shows the influence of the applied pressure in the stress state of the plate as the temperature increases. One can see a redistribution in the combined stress state of the overall plate area when the temperature increases from 100 to $400^{\circ} \mathrm{C}$. It can also be seen that the maximum value of the combined stress does not increase from 100 to $400^{\circ} \mathrm{C}$. In fact increasing the temperature will induce additional compressive loads to the plate which are compensated with the decrease of the average compressive stress, as result of the increasing curvature, due to the lateral pressure.

Figs. 15-19 to plot the results grouped by the plate slenderness and showing the variation of the plate behaviour for the three levels of lateral pressure, i.e., 0,10 and 50 $\mathrm{m}$ of water height.

One may conclude that normal levels of lateral pressure (i.e., up to $10 \mathrm{~m}$ water depth) do not change the form of the average stress-temperature curve of the square plate and, thus, the curve with lateral pressure may be estimated from the ones without pressure by introducing a correction factor. The gap between these two curves $(0$ and $0.1 \mathrm{MPa})$ in each graph increases with the $b / t$ ratio, which is in accordance with the increase in the non-dimensional lateral pressure parameter with the same ratio, if one includes the plate geometry in this parameter (as assumed in Section 5 below).

\subsection{Behaviour of rectangular plates}

The effect of lateral pressure on rectangular plates of aspect ratio of 3 with increasing temperature was investigated. The imposed initial imperfections have one

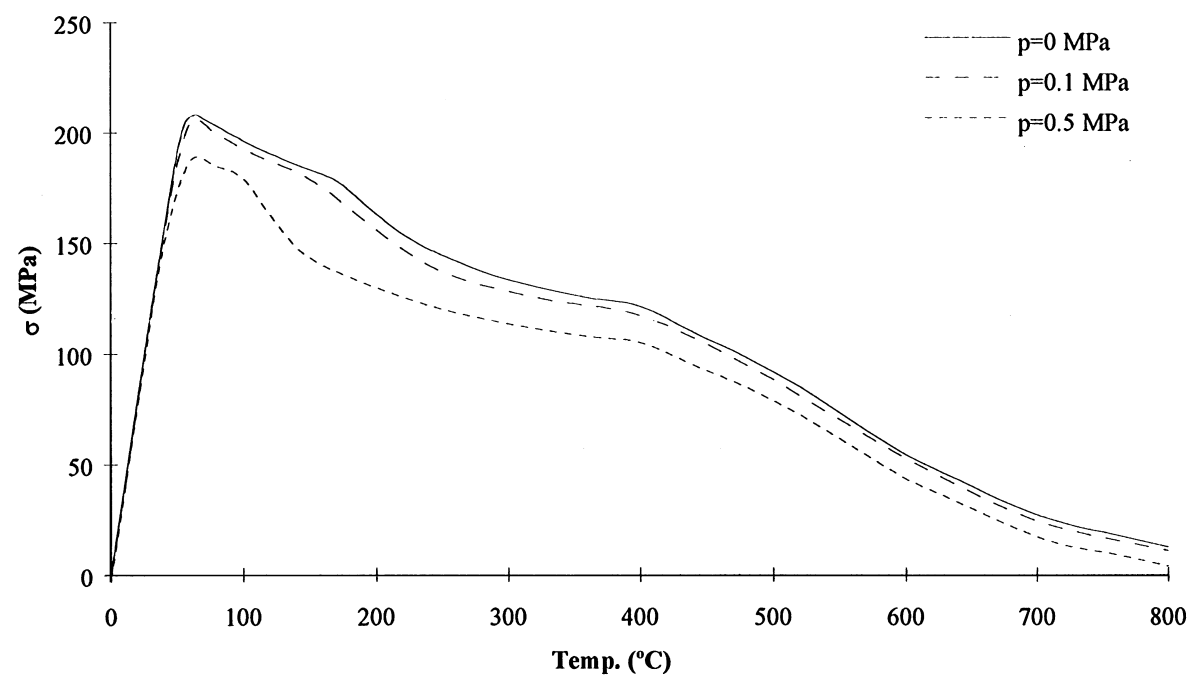

Fig. 15. Behaviour of square plates under temperature with applied lateral pressure. Effect of increasing lateral pressure on plates of $b / t=20$. 


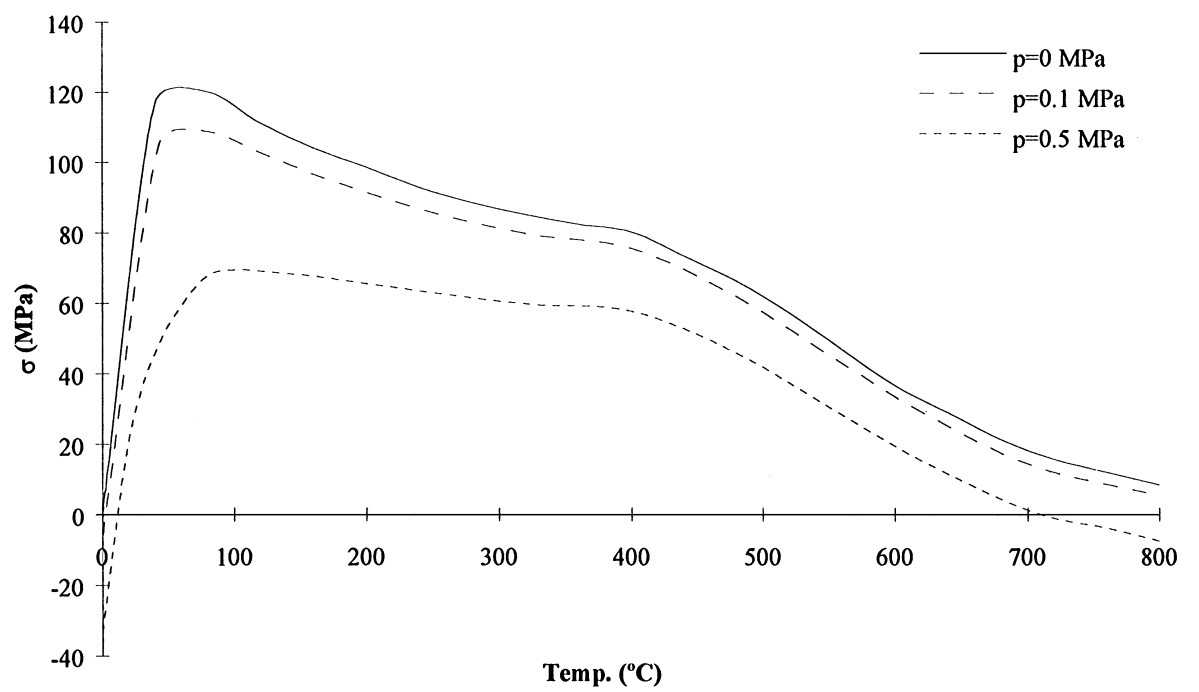

Fig. 16. Behaviour of square plates under temperature with applied lateral pressure. Effect of increasing lateral pressure on plates of $b / t=40$.

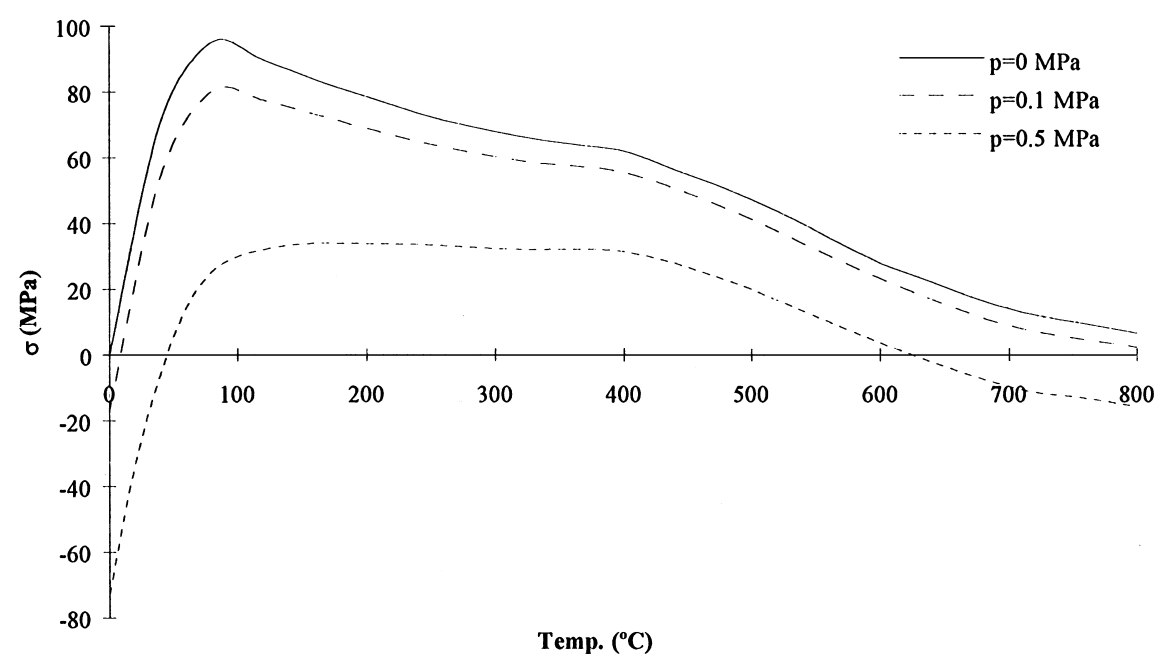

Fig. 17. Behaviour of square plates under temperature with applied lateral pressure. Effect of increasing lateral pressure on plates of $b / t=60$.

half-wave shape, which is the same shape of the deformations due to lateral pressure. This imposed mode associated with the lateral pressure tends to increase the strength in the longitudinal direction and to reduce it in the transverse direction.

Figs. 20 and 21 show the longitudinal average stress-temperature curves for 0.1 and $0.5 \mathrm{MPa}$ of lateral pressure. A reduction may be noticed in the maximum stress and 


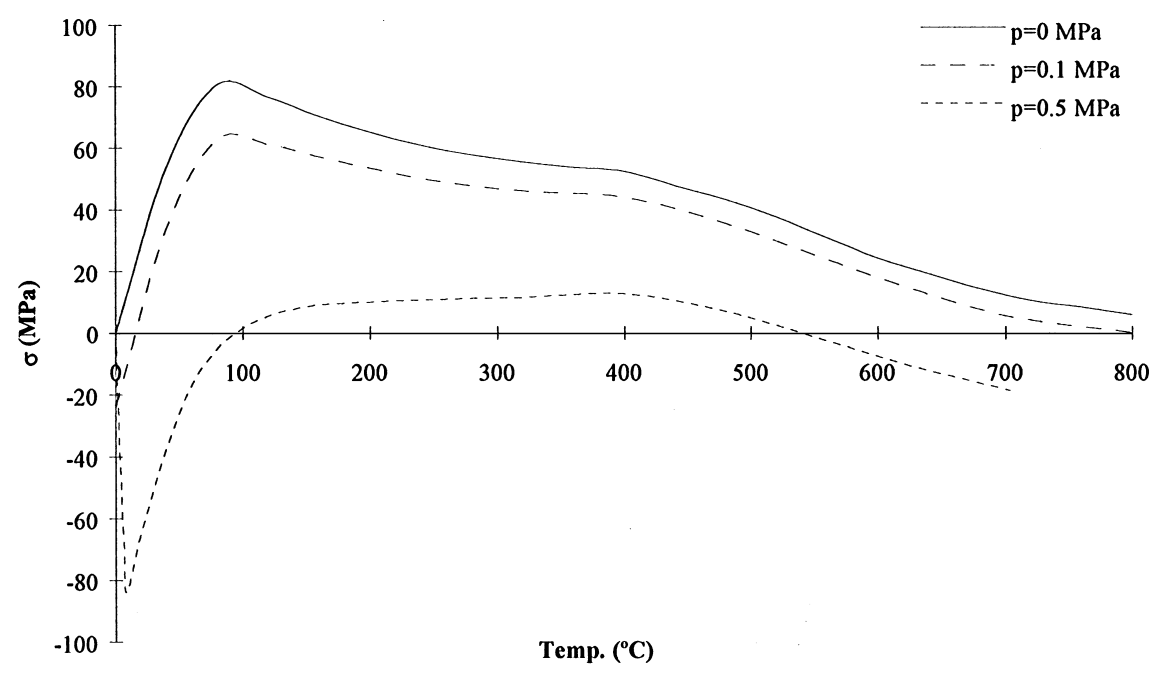

Fig. 18. Behaviour of square plates under temperature with applied lateral pressure. Effect of increasing lateral pressure on plates of $b / t=80$.

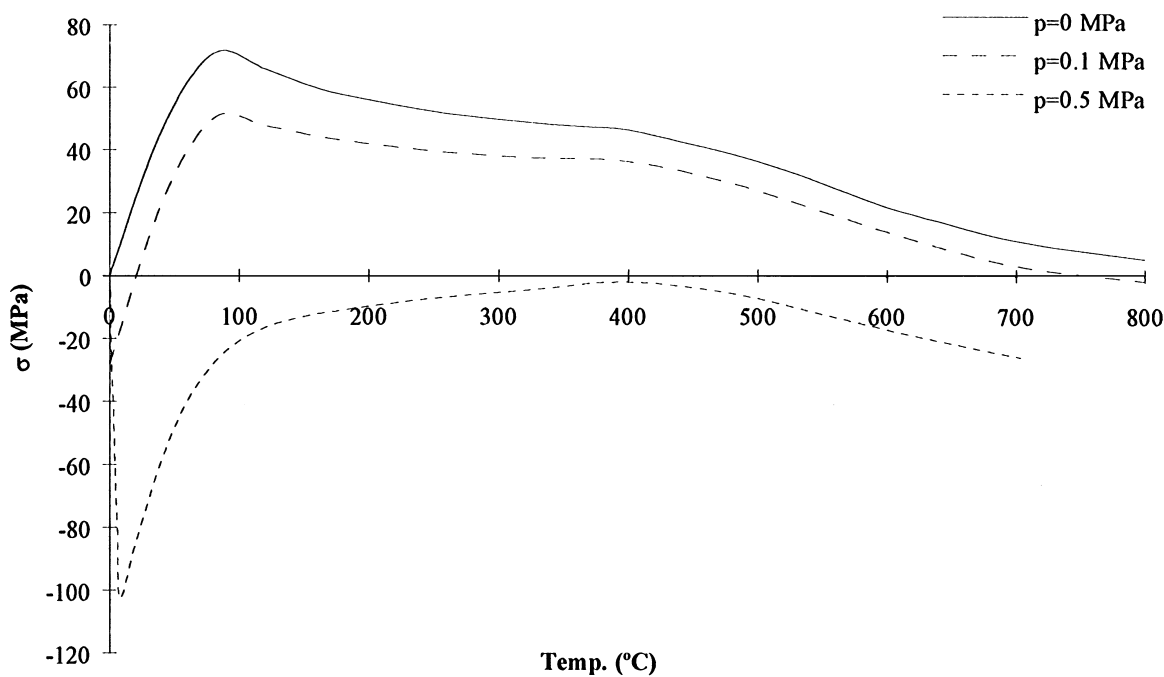

Fig. 19. Behaviour of square plates under temperature with applied lateral pressure. Effect of increasing lateral pressure on plates of $b / t=100$.

the curves tend to be smoother when the pressure increases. For the highest level of pressure the longitudinal stress recorded in the range of $200-400^{\circ} \mathrm{C}$ is almost constant.

At very high temperature and high slenderness the lateral pressure is mainly supported by tension stresses, i.e., the very high deformations of the plate is due to the 


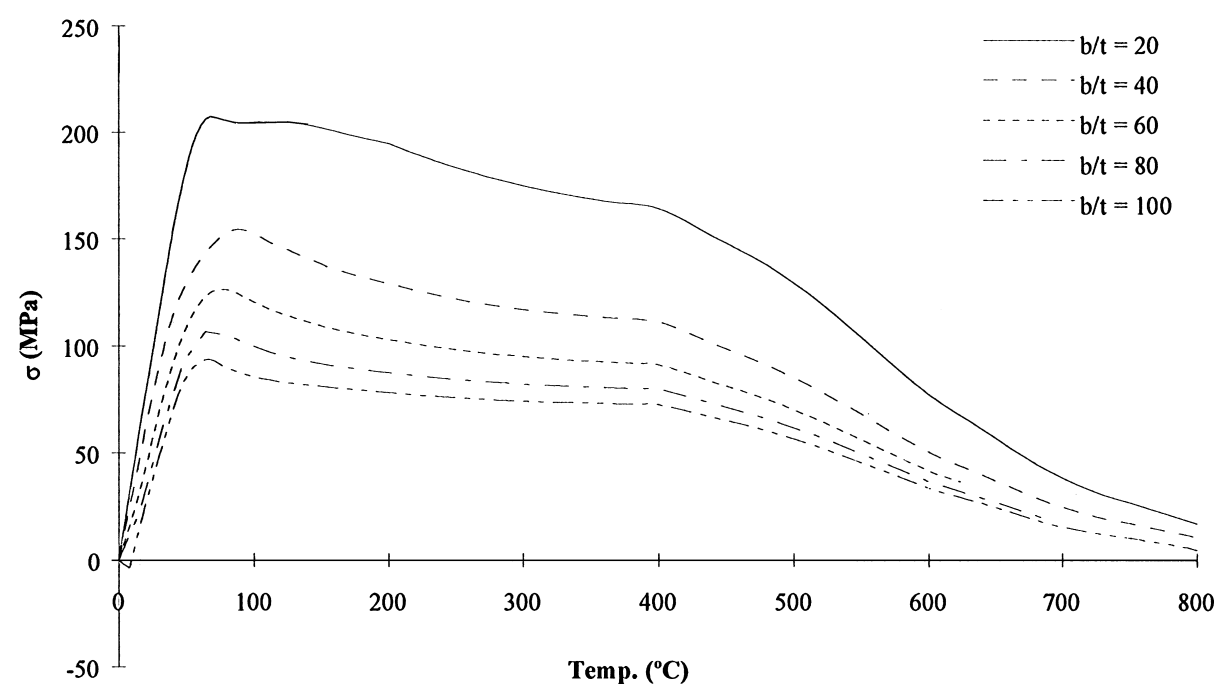

Fig. 20. Behaviour of rectangular plates $(a / b=3)$ under temperature with applied lateral pressure of $0.1 \mathrm{MPa}$. Stress refers to longitudinal direction.

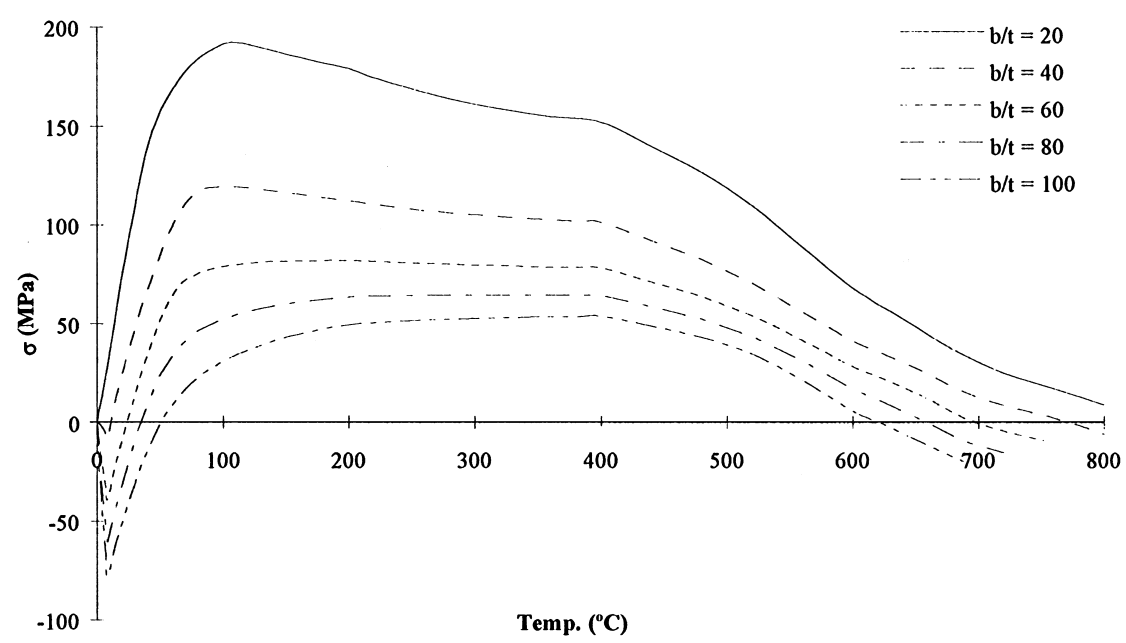

Fig. 21. Behaviour of rectangular plates $(a / b=3)$ under temperature with applied lateral pressure of $0.5 \mathrm{MPa}$. Stresses refer to longitudinal direction.

applied pressure and the low material yield stress at that temperature compensate largely for the expansion of the plate due to the temperature difference.

Figs. 22-24 compare the stress levels in the longitudinal and transverse directions. For $b / t=20$ plates, first collapse is achieved in the transverse direction at very low temperatures (approximately $50^{\circ} \mathrm{C}$ ), and it is followed by a rapid decrease in the load-carrying capacity in this direction. 


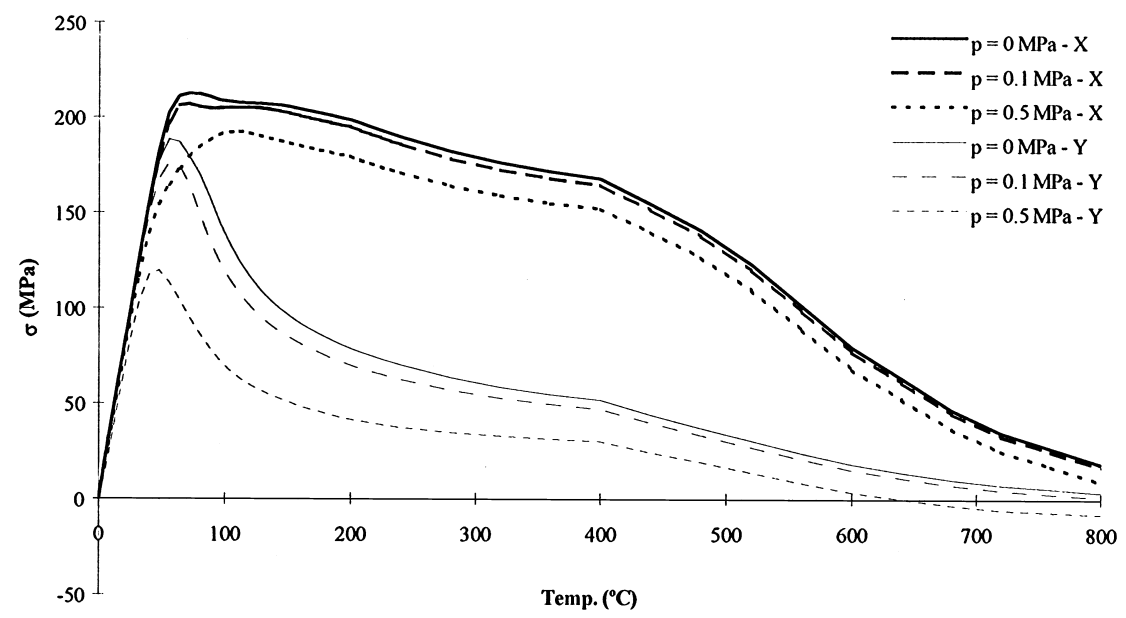

Fig. 22. Behaviour of rectangular plates $(a / b=3)$ under temperature with applied lateral pressure. Effect of increasing lateral pressure on plates of $b / t=20 . X$ represents longitudinal stresses and $Y$ transverse stresses.

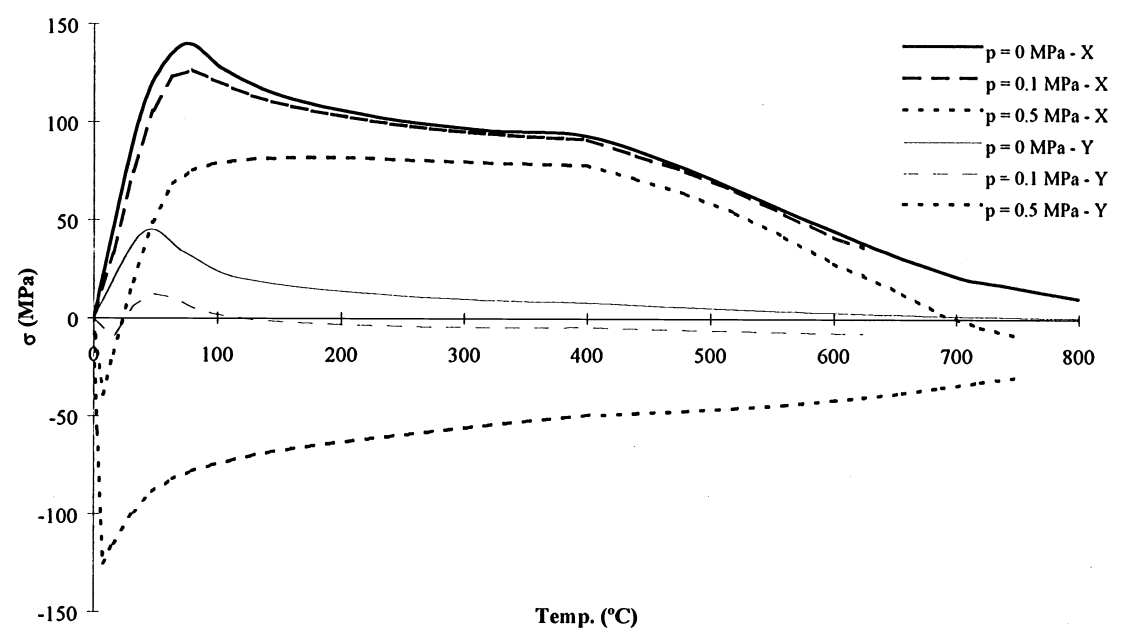

Fig. 23. Behaviour of rectangular plates $(a / b=3)$ under temperature with applied lateral pressure. Effect of increasing lateral pressure on plates of $b / t=60 . X$ represents longitudinal stresses and $Y$ transverse stresses.

For plates of $b / t$ greater than 40 the transverse stress tends quickly to zero, once again, which means, that the expansion in the transverse direction is converted in out-of-plane deformations helped by the work done by the lateral pressure. At very high pressure and moderate to high slenderness, the plate is always in tension in the transverse direction.

The shape of initial imperfections chosen for this analysis gives results that are a lower bound of strength. High modes of imperfections, especially those that have the 


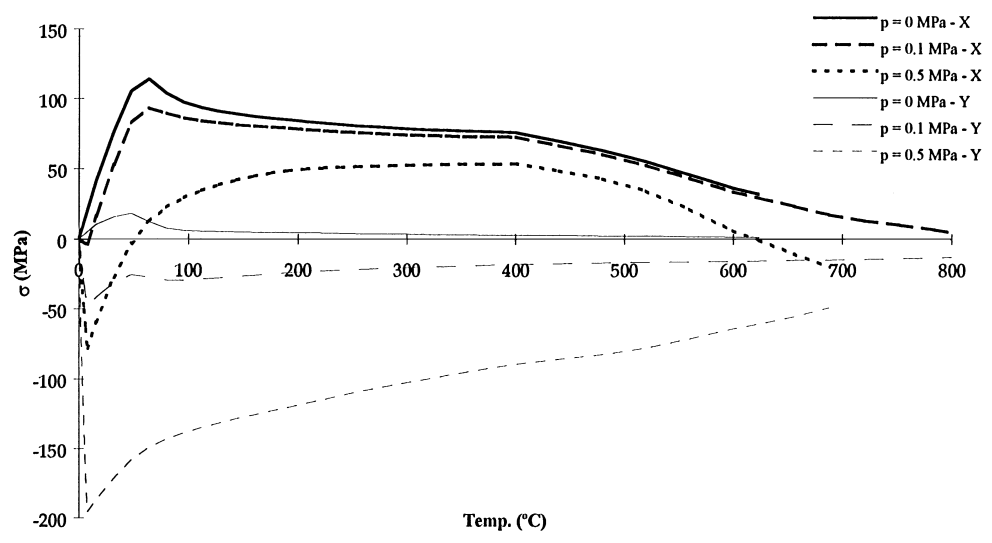

Fig. 24. Behaviour of rectangular plates $(a / b=3)$ under temperature with applied lateral pressure. Effect of increasing lateral pressure on plates of $b / t=100 . X$ represents longitudinal stresses and $\mathrm{Y}$ transverse stresses.

same number of half-waves as the aspect ratio, change the transverse stresses to levels close to the longitudinal ones, especially in those cases, where lateral pressure is kept at low values. Thus, in this case one may use the square plate behaviour to model the rectangular one. However, the stochastic nature of the imperfections recommends the use of this lower bound because the first mode of initial geometric imperfections is predominant in 'normal' plates in service due to residual stresses and the effect of lateral pressure.

\section{Interaction curves for plates with lateral ressure}

In order to estimate the reduction in the plate strength due to the presence of lateral pressure, one needs to normalise the pressure. Dier and Dowling [11] have considered that the lateral pressure could be represented by the non-dimensional parameter

$$
Q_{l}=\frac{p E}{\sigma_{o}^{2}}
$$

where $p$ is the intensity of the lateral pressure. Using this parameter the range of the lateral pressure considered in this study varies from 0.38 to 1.9 .

Square plates have the same stress in both directions at a given temperature, thus it is sufficient to compute the degradation in strength in one direction, and extrapolate the results of $R$ as given by Eq. (8).

Figs. 25 and 26 show for square plates the ratio between the stress with applied lateral pressure and the corresponding stress without lateral pressure. 


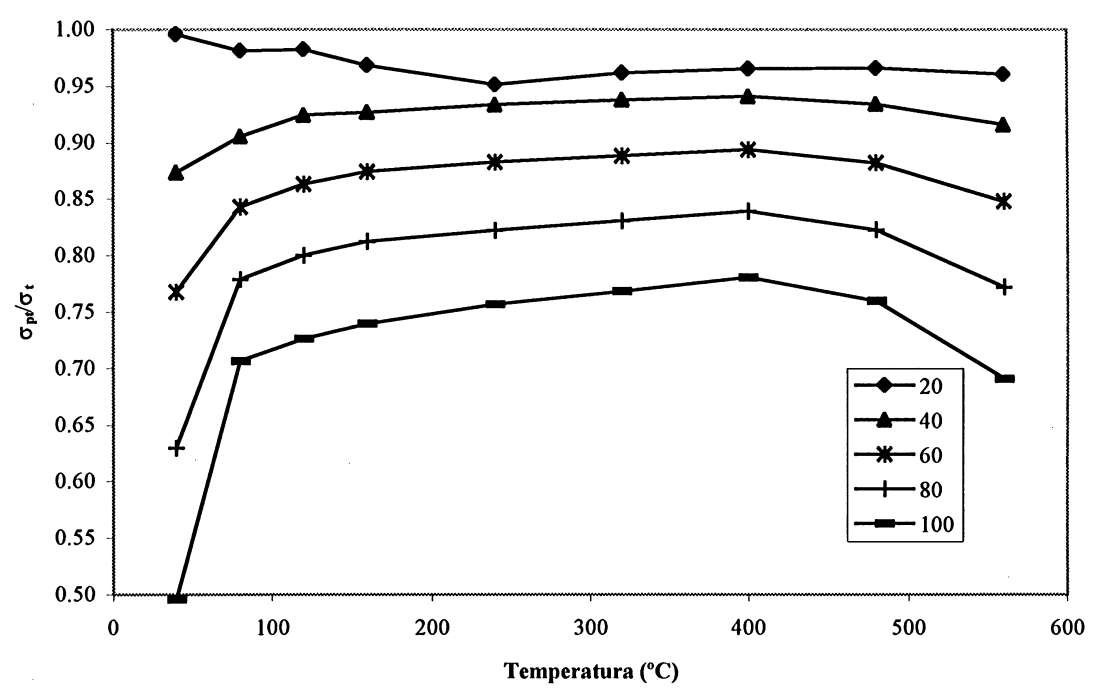

Fig. 25. Ratio between the strength of square plate with $\left(\sigma_{p t}\right)$ and without $\left(\sigma_{t}\right)$ lateral pressure for $p=0.1 \mathrm{MPa}$ and several plate slenderness.

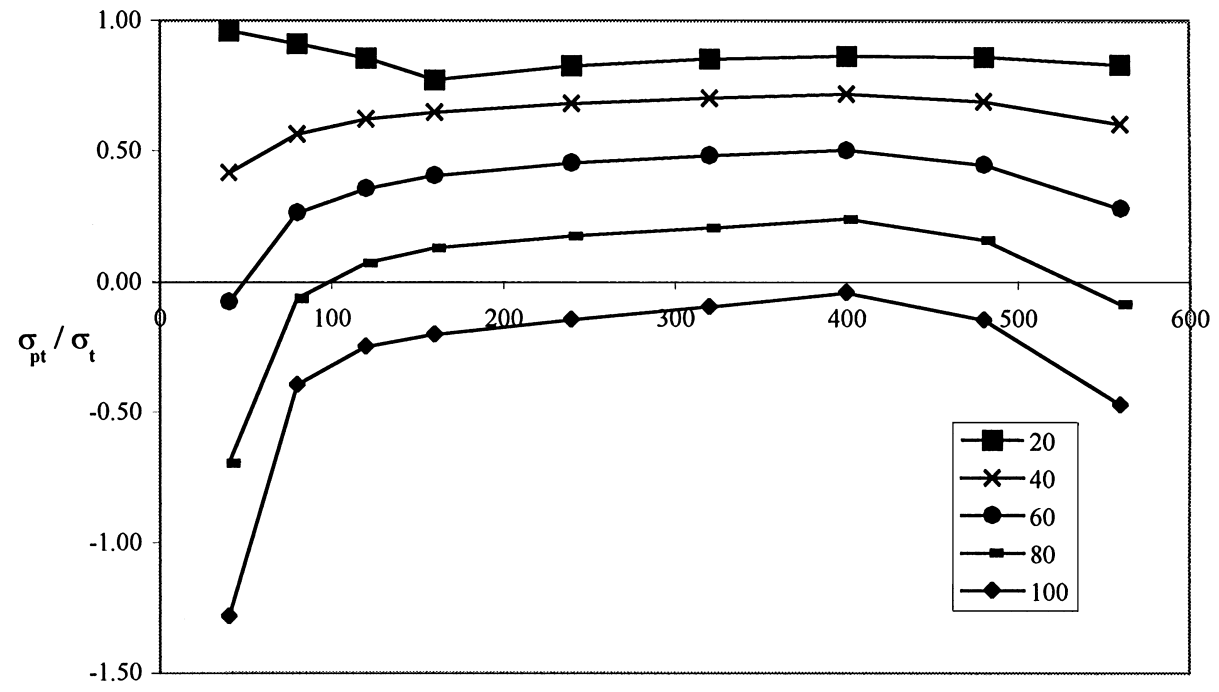

Temperatura $\left({ }^{\circ} \mathrm{C}\right)$

Fig. 26. Ratio between the strength of square plate with $\left(\sigma_{p t}\right)$ and without lateral pressure $\left(\sigma_{t}\right)$ for $p=0.5 \mathrm{MPa}$ and several plate slenderness.

The ratios are approximately constant with the increase in temperature and all curves show the same type of behaviour. In the plastic range $\left(T>200^{\circ} \mathrm{C}\right)$ the curves increase only a small amount until $400^{\circ} \mathrm{C}$, reducing the degradation of the strength, and after that point they decrease. The shape of the curves seems to be similar to that 
implicit in the curve of the ultimate stress, Eq. (15), associated with the average strain. However, this behaviour was not considered because the variation is not high in the range $160-560^{\circ} \mathrm{C}$.

The main variation is associated with the level of the lateral pressure and with the width to thickness ratio of the plate. The degradation is linear with respect to lateral pressure, in the whole range considered, which is a significant one since the highest pressure is $50 \mathrm{~m}$ of water.

The correlation between reduction in strength and initial plate slenderness, at normal temperature, is somewhere between linear and quadratic, but the coefficients of correlation does not vary much.

For design purposes the quadratic dependence was adopted as the most appropriate one and the design stress may be expressed by:

$$
\sigma_{p t}=\sigma_{t} \cdot\left(1-0.057 \beta^{2} Q_{l}\right)
$$

where $\sigma_{t}$ is the ultimate carrying capacity of a square plate under temperature, and $\beta$ is calculated with the initial properties of the material corresponding to ambient temperature. The regression demonstrated a correlation coefficient of 0.975. An even better correlation may be obtained if the ultimate tensile stress is adopted for the stress ratios instead of the yield stress.

However, a large difference exists between $\sigma_{t}$ and $\sigma_{p t}$. While $\sigma_{t}$ represents the ultimate carrying capacity of a square plate under temperature, $\sigma_{p t}$ is the actual state of in-plane stress under lateral pressure and temperature for fixed simply supported boundary conditions.

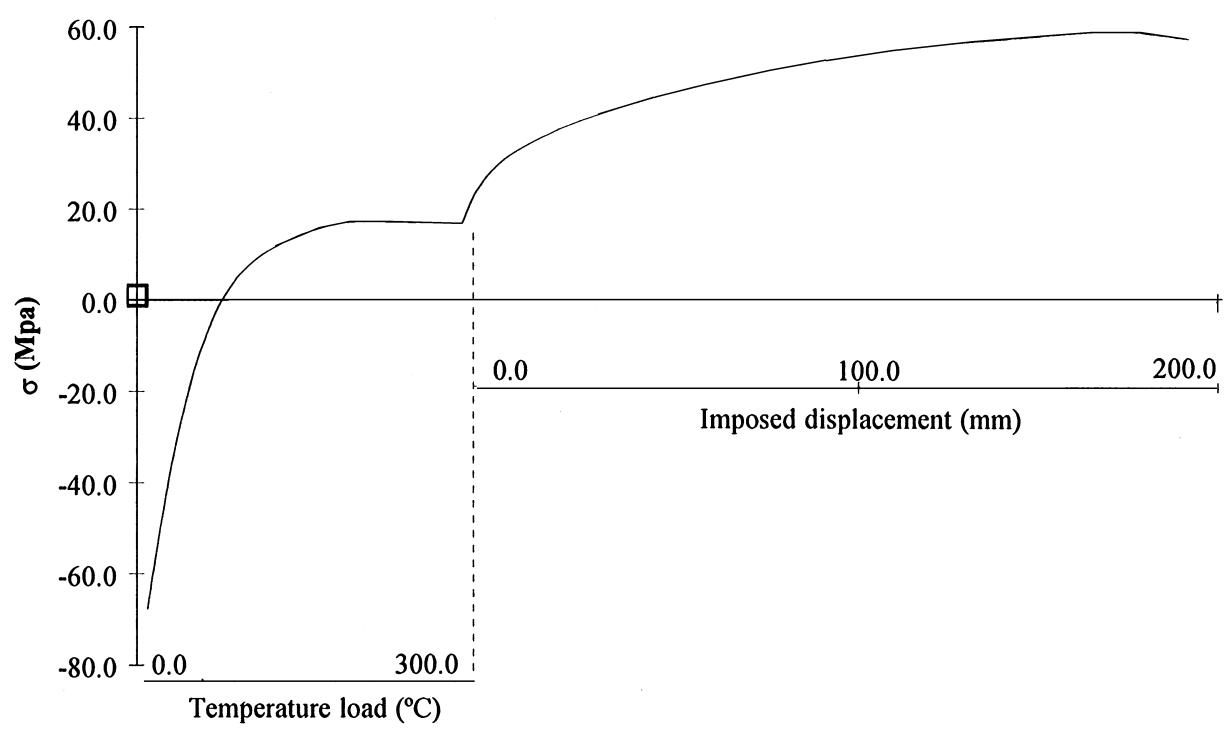

Fig. 27. Behaviour of a square plate $(b / t=60)$ with imposed displacement to one edge after lateral pressure and temperature loads. 
Under these states of stress and deformations the plate still has some capability to sustain additional load. This have been proved by applying three different loading to a square plate of $b / t=60$ : first, the lateral pressure, then increasing the temperature to $300^{\circ} \mathrm{C}$ and, after that, imposing displacements to one edge. The results are summarised in Fig. 27 showing that the plate may sustain more than the double of the actual stress $\sigma_{p t}$, but with a very large shortening. For this case the average nominal shortening is almost $10 \%$.

\section{Conclusions}

This parametric study has determined load-temperature curves for plates ranging from $a / b=1$ to 3 and from $b / t=20$ to 100 with different levels and shapes of initial distortions.

It was observed that the maximum load-carrying capacity of the plates is often in the temperature range from 100 to $200^{\circ} \mathrm{C}$, a region in which the yield stress of the material has yet not decreased significantly.

The maximum load-carrying capability is adequately described by the circular interaction relation applicable to plates at ambient temperature.

The strength of the plate at high temperatures is also described accurately by the circular interaction formula if an appropriate choice of the reference stress is made. The reference stress to non-dimensionalise the collapse stresses of the plates in both directions should be the average stress estimated by interpolation between the ultimate tensile and the yield stress of the material at the respective temperature. Using the yield stress will underestimate the strength of the plate, thus providing a low, bound to the carrying capacity of the plate.

The effect of heat loads on plates subjected to lateral pressure was studied. It was shown that normal levels of lateral pressure (i.e., up to $10 \mathrm{~m}$ water height) do not change the form of the average stress-temperature curve of the plate and, thus, the curves with lateral pressure may be estimated from the ones without pressure by introducing a correction factor. This fact presented an opportunity to define interaction curves for square plates to estimate the reduction in plate strength when initially subjected to lateral pressure.

\section{Acknowledgements}

This study has been performed in the project "Optimised Fire Safety of Offshore Structures (OFSOS)" which has been partially funded by the European Commission under the BRITE/EURAM program, under contract $\mathrm{n}^{\circ}$ 92/0598. The other partners of this project where Registro Italiano Navale, AEA Petroleum Service, Computational Safety and Reliability, Germanischer Lloyd, SNAMPROGETTI, TECNOMARE, and WS Atkins, having as sponsors AGIP, British Gas, Chevron and Amoco. 


\section{References}

[1] Guedes Soares C, Gordo JM, Teixeira AP. Elasto- plastic behaviour of plates subjected to heat loads. J Construct Steel Res 1998;45(2):179-98.

[2] Guedes Soares C. Mechanical properties of steel at elevated temperatures. OFSOS Report TECC021-02.

[3] Commission for the European Communities. Eurocode n.3 (EC3): design of steel structures. Structural fire design, part 10, 1990.

[4] Guedes Soares C. Design equation for the compressive strength of unstiffened plate elements with initial imperfections. Construct Steel Res 1988;9:287-310.

[5] ASASNL, Version 19. England, WS Atkins Engineering Sciences Lda, 1990.

[6] Faulkner D. A review of effective plating for use in the analysis of stiffened plating in bending and compression. Ship Res, 1975;19:1-17.

[7] Guedes Soares C, Faulkner D. Probabilistic modelling of the effect of initial imperfections on the compressive strength of rectangular plates. Proceedings of 3rd International Symposium on Practical Design of Ships and Mobile Units (PRADS). Trondheim 1987;2:783-95.

[8] Valsgard S. Numerical design prediction of the capacity of plates in in-plane compression. Comput Struct 1980;12:729-39.

[9] Faulkner D, Adamchak JC, Snyder GJ, Vetter MF. Synthesis of welded grillages to withstand compression and normal loads. Comput Struct 1973;3:221-46.

[10] Guedes Soares C, Gordo JM. Compressive strength of rectangular plates under biaxial load and lateral pressure, Thin-walled structures, vol. 22, Amsterdam, Elsevier Science, 1996, p. 231-59.

[11] Dier AF. Dowling PJ. Plates under combined lateral loading and biaxial compression. CESLIC Report SP8, Imperial College, Department of Civil Engineering London, 1980. 